\title{
碱性燃料电池用聚烯烃类阴离子交换膜的研究进展
}

\author{
刘磊，褚晓萌，李南文* \\ 中国科学院山西煤炭化学研究所, 煤转化国家重点实验室, 太原 030001 \\ *联系人, E-mail: linanwen@sxicc.ac.cn
}

2018-08-15 收稿, 2018-09-14 修回, 2018-09-17 接受, 2018-10-31 网络版发表

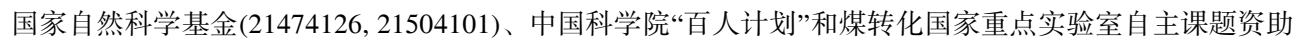

\begin{abstract}
摘要与质子交换膜燃料电池相比, 基于阴离子交换膜的碱性燃料电池具有可使用非贵金属催化剂、电极反应 速率高等优点，近年来受到广泛的关注。然而，到目前为止，尚未开发出一种高性能的阴离子交换膜以备碱性燃 料电池使用. 本文从功能单体共聚和高分子接枝改性两方面概述了聚烯烃类阴离子交换膜的制备方法, 探讨了膜 的化学结构、微观相分离结构与膜性能之间关系, 最后总结目前聚烯烃类阴离子交换膜的氢氧燃料电池性能, 并 对该领域的发展趋势进行了展望.
\end{abstract}

关键词聚烯烃，阴离子交换膜，碱性燃料电池，电导率，碱性稳定性

阴离子交换膜燃料电池结合了质子交换膜燃料 电池和碱性燃料电池的优点. 与质子交换膜燃料电 池相比，阴离子交换膜燃料电池的优势在于：在碱性 介质中, 氧气还原的动力学优于酸性介质中; 氢氧根 离子的迁移方向与甲醇在膜中的渗透方向相反, 能 够有效降低甲醇的透过; 催化剂在碱性介质下的选 择范围大大拓宽, 能够使用便宜的非贵金属, 如Fe, $\mathrm{Ni}, \mathrm{Ag}$ 等. 此外, 与传统的碱性燃料电池相比, 采用 固态的聚合物电解质能够有效地避免液体电解质的 泄露和碳酸盐化, 提高燃料电池的使用寿命 ${ }^{[1 ~ 3]}$.

聚合物膜材料是阴离子交换膜燃料电池最重要 的部件之一, 它一方面起着氢氧根离子传导的功能, 另一方面阻隔燃料和氧化剂, 防止燃料渗透而造成 电池内部短路. 因此, 作为一个理想的燃料电池用的 阴离子交换膜, 它需要具有良好的离子传导率、优异 的机械性能和化学稳定性. 但是, 与质子交换膜(如 Nafion)相比, 阴离子交换膜在离子传导率和化学稳 定性方面还有需要进一步提高, 主要原因在于: (1) 在碱性条件下, 尤其是在较高温度下 $\left(>60^{\circ} \mathrm{C}\right)$, 阴离
子交换膜的化学结构容易发生降解；(2) 氢氧根离子 在无限稀释的水溶液中的迁移率低于质子迁移率 ${ }^{[4,5]}$. 从化学结构来看, 阴离子交换膜是指聚合物上 固定有阴离子交换基团(如季铵盐)的膜. 因此，阴离 子交换膜的化学组成包括两个部分: (1) 聚合物主链, 主要提供膜的机械性能;（2）阳离子基团，提供阴离 子传导的功能. 常见的用于阴离子交换膜的阳离子 基团如图 1所示，主要可以分为两大类：有机阳离子 基团和金属配合物阳离子基团. 由于阴离子交换膜 燃料电池中强碱的工作环境，阳离子基团容易受到 氢氧根离子的进攻而发生降解，导致膜的性能下降， 其中，降解机理包括消除反应和亲核取代反应等 ${ }^{[6]}$. Marino等人 ${ }^{[7]}$ 和Mohanty等人 ${ }^{[8]}$ 详细研究了不同阳离 子基团的碱性稳定性，发现具有螺环结构和长烷基 链取代的阳离子基团在碱性条件下稳定性较高. 虽 然不同种类的阳离子基团都被用于阴离子交换膜， 但是季铵盐基团是目前研究最广泛的, 这主要是由 于其合成方法比较简单、成本较低.

为了提供阴离子交换膜优异的机械性能，芳族

引用格式：刘否，褚晓萌，李南文. 碱性燃料电池用聚烯烃类阴离子交换膜的研究进展. 科学通报, 2019, 64: 123-133 Liu L, Chu X M, Li N W. Recent development in polyolefin-based anion exchange membrane for fuel cell application (in Chinese). Chin Sci Bull, 2019, 64: 123-133, doi: 10.1360/N972018-00880 


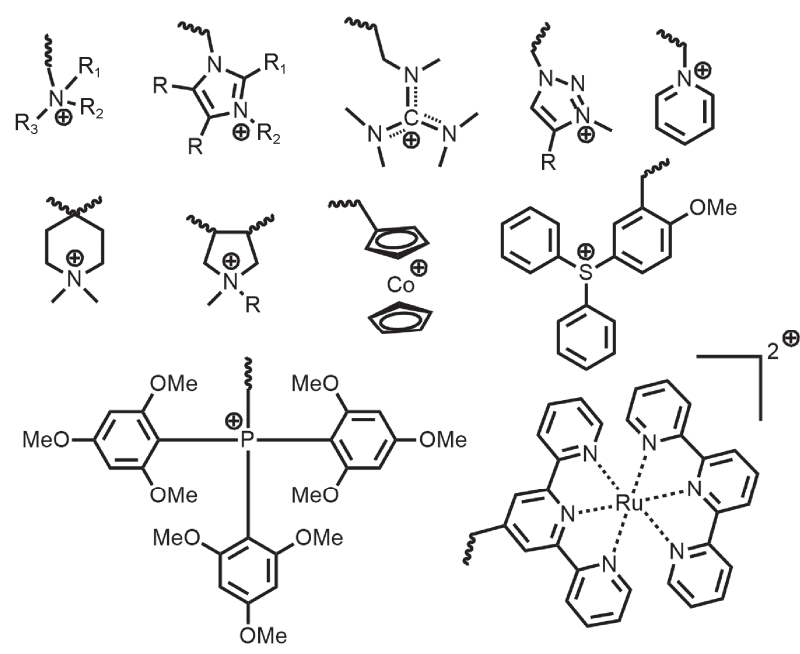

图 1 阴离子交换膜(AEMs)常用的阳离子基团

Figure 1 Organic cations in AEMs

聚合物和聚烯烃类聚合物常被用作阴离子交换膜的 聚合物主链材料, 其中, 芳族聚合物包括聚醚 砜 $^{[9,10]}$ 、聚苯醚 ${ }^{[11]} 、$ 聚醚醚酮 ${ }^{[12]}$, 聚苯并咪唑 ${ }^{[13]}$ 等. 但 是, 季铵化的芳族聚合物主链 (尤其是聚醚砜类聚合 物)在碱性条件下容易受到氢氧根离子的进攻而发生 降解反应, 导致膜失去机械性能而不能使用 ${ }^{[14,15]}$. 与 芳族聚合物比, 聚烯烃作为阴离子交换膜的骨架材 料具有化学稳定性高、加工性能好和价格低廉的优 点. 但是聚烯烃阴离子交换膜材料合成比较困难, 研 究者近年来才开始关注. 因此, 本文从聚烯烃类阴离 子交换膜的制备方法、性能和氢氧燃料电池性能 3 个 方面出发, 对近年来的研究工作进行总结和梳理.

\section{1 聚烯烃类阴离子交换膜的制备方法}

\section{1 功能单体共聚}

由于阴离子交换膜的主链是聚烯烃类聚合物, 因此, 所采用的功能单体大部分是一些非共轭的乙 烯基单体, 如图2所示.

近年来, 降冰片烯类和环辛烯类是研究最多的 功能单体, 如图2中的 1 8. 通过开环易位聚合反应 (ROMP), 得到主链是聚烯烃的阴离子交换膜材料. Coates课题组 ${ }^{[16]}$ 通过 D-A 反应, 设计合成不含有 $\beta-\mathrm{H}$ 的季铵盐型降冰片烯功能单体 1 , 并且利用 Grubbs二 代催化剂, 进行功能单体 1 与双环戊二烯进行开环易 位聚合, 得到了一种交联的阴离子交换膜. 膜的电导 率测试结果表明, 当膜的离子交换容量(IEC)为 1.0 $\mathrm{mmol} / \mathrm{g}$ 时, $20^{\circ} \mathrm{C}$ 时的氢氧根离子电导率为 $14 \mathrm{mS} / \mathrm{cm}$, 甲醇中的溶胀率为 $1.7 \%$, 膜的拉伸强度达到 $16 \mathrm{MPa}$. Coates课题组 ${ }^{[17]}$ 随后利用季铵盐型环辛烯类功能单 体 3, 制备了交联的聚烯烃类阴离子交换膜, 并且膜 具有较高的电导率. Zha等人 ${ }^{[18]}$ 设计合成了一种二价 的降冰片烯类钉金属离子功能单体 $\mathbf{8}$, 并通过与双环 戊二烯的开环易位聚合, 得到交联的金属离子聚烯 烃类阴离子交换膜. 然而, ROMP方法得到的聚烯烃 聚合物主链上含有大量双键, 导致膜的主链结构在 碱性条件下稳定性较差. 因此, 为了制备碱稳定的阴 离子交换膜材料, Coates课题组 ${ }^{[19 \sim 21]}$ 在随后的工作 中, 采用金属催化加氢的方法, 得到有机溶剂可溶的 聚烯烃阴离子交换膜材料, 如图 2(1a)所示. Zhu等 人 ${ }^{[22]}$ 采用同样的方法, 得到含有二茂钴阳离子的可 溶的聚烯烃类阴离子交换膜材料.

$\alpha$-烯烃功能单体的直接聚合是制备聚烯烃类离 子交换膜的重要方法之一, 但是传统的自由基聚合 反应无法得到高分子量、结构明确的聚烯烃离子交换 膜, 并且利用Ziegler-Natta催化剂直接进行 $\alpha$-烯烃功 能单体与烯烃的共聚反应容易导致催化剂的毒化. Zhang等人 ${ }^{[23]}$ 采用硅烷保护的胺基 $\alpha$-烯烃功能单体 9 , 在茂金属络合物催化下，与乙烯进行共聚，得到高分 子量的聚乙烯类聚合物, 再通过官能团的转化, 得到 季铵盐型聚乙烯阴离子交换膜. 然而, 该类方法得到 的聚烯烃功能单体插人率较低(小于 $10 \%$ ). 本课题 组 ${ }^{[24]}$ 采用Ziegler-Natta催化剂进行11-溴-1-十一碳烯 (功能单体 10) 与丙烯在密闭高压釜中共聚, 含溴功能 单体的插人率可高达 $20 \mathrm{~mol} \%$, 并且得到的聚烯烃的 重均分子量达到 129 386 kg/mol, 分子量分布6.1 7.0. 再经过与三甲胺进行季铵化反应, 得到聚烯烃 类阴离子交换膜, 反应过程如图2(b)所示 ${ }^{[24]}$. 进一步 改变疏水端的化学结构, 例如, 功能单体 10分别与4甲基-1-戊烯(PMP $)^{[25]}$ 及 4-苯基-1-丁烯(P4BP $)^{[26]}$ 共聚, 不但可以实现常压下聚合, 而且能够得到高分子量 的聚烯烃阴离子交换膜材料. 然而, 所得到的聚烯烃 无法在有机溶剂中溶解, 只能通过热压成膜. 此外, 由于适用的功能单体较少, 采用 $\alpha$-烯烃直接聚合制 备聚烯烃类阴离子交换膜受到了一定的限制.

二烯丙基二烷基季铵盐是一种特殊的季铵盐型 功能单体. 通过相邻的 2 个烯丙基的自由基聚合, 容 易得到分子内成环的线性聚合物. $\mathrm{Gu}$ 等人 ${ }^{[27]}$ 通过二 烯丙基甲基乙基季铵盐(功能单体 12)与丙烯腈、苯乙 
1. Copolymerization

(a) Ring-opening metathesis polymerization

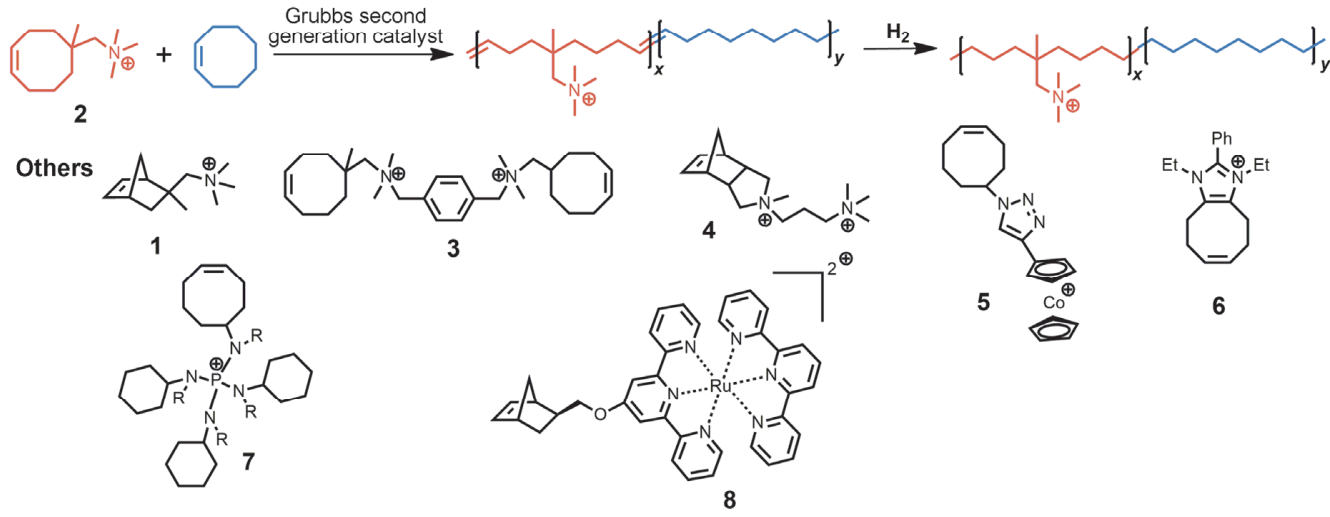

(b) Copolymerization with Ziegler-Natta catalysts

(c) Radical copolymerization
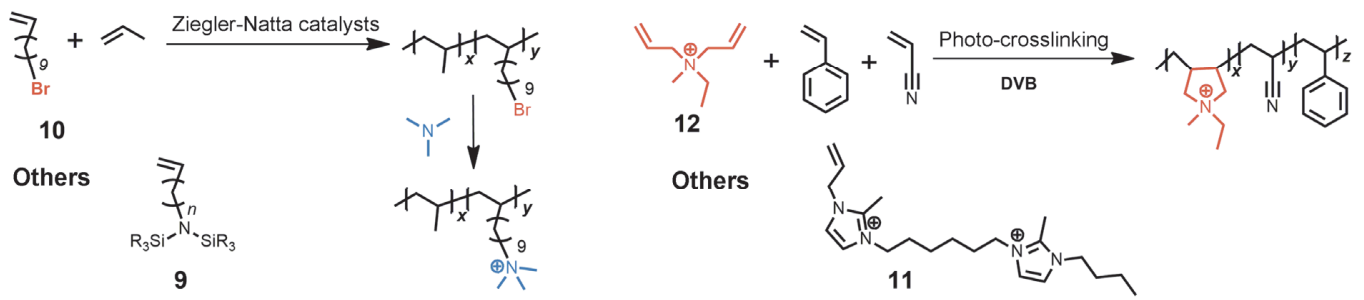

2. Radiation grafting

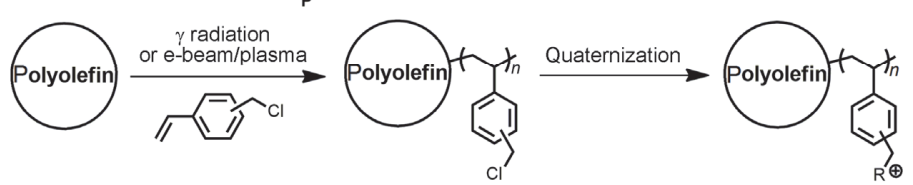

图 2 (网络版彩色)聚烯烃类阴离子交换膜的制备

Figure 2 (Color online) Preparation of AEMs based on polyolefins

烯的光交联聚合反应, 得到吡咯盐型阴离子交换膜. 采用同样的方法, Qiu等人 ${ }^{[28]}$ 利用双咪唑盐功能单体 11, 得到咪唑盐型阴离子交换膜, 但是该类烯丙基单 体难以实现可控的聚合反应, 无法得到IEC值可控的 阴离子交换膜.

\section{2 高分子接枝}

接枝改性是聚合物功能化的一种重要方法, 具 有操作简单、高效的特点. 目前, 辐射接枝是制备聚 烯烃类阴离子交换膜的最常用方法, 通过辐射的手 段在聚烯烃主链上接枝聚合对氯甲基苯乙烯单体, 再经季铵化反应, 得到聚烯烃类阴离子交换膜, 反应 路线如图2所示.

在高能射线辐照下 $\left({ }^{60} \mathrm{Co} \gamma\right.$ 射线或者电子束), 聚 烯烃材料表面产生活性位点: 自由基或者过氧化物, 再进一步与对氯甲基苯乙烯进行接枝聚合反应, 得
到功能化的聚烯烃. Danks等人 ${ }^{[29]}$ 将 $\gamma$ 射线辐照处理 的聚偏氟乙烯(PVDF) 和四氟乙烯-六氟丙烯共聚物 (FEP) 膜浸人对氯甲基苯乙烯中，分别得到接枝率为 $54 \%$ 和 $27 \%$ 的接枝聚合物膜, 再浸人三甲胺溶液中, 得到聚偏氟乙烯和四氟乙烯-六氟丙烯共聚物阴离子 交换膜. Liu等人 ${ }^{[30]}$ 和Fang等人 ${ }^{[31]}$ 采用同样的方法, 分别得到四氟乙烯-全氟乙烯基醚(PFA)和乙烯-四氟 乙烯共聚物(ETFE)阴离子交换膜. Mamlouk等人 ${ }^{[32]}$ 比 较了三种聚烯烃(ETFE, 低密度聚乙烯和高密度聚乙 烯)的辐射接枝条件与阴离子交换膜性能之间的关 系, 研究结果表明, 全氟或者部分氟化的聚合物需要 更高的辐射剂量才能得到与碳氢聚合物类相当的接 枝率, 并且高接枝率有利于提高膜的电导率. 为了提 高辐射接枝阴离子交换膜的电导率，人们通常采用 增加辐射剂量的方法, 但是高的辐射剂量容易导致 膜的机械性能急剧下降. Wang等人 ${ }^{[33]}$ 在空气中用电 
子束分别对低密度聚乙烯和ETFE进行预处理, 使得 聚乙烯膜表面产生过氧化物官能团, 再进行接枝聚 合反应和季铵化反应, 得到高性能的低密度聚乙烯 阴离子交换膜. 值得一提的是, 低密度聚乙烯膜在辐 射后, 其机械性能未发生明显变化, 而ETFE膜的机 械性能下降较多, 这主要是由于当辐射剂量超过 10 $k G y$ 时, 部分氟化的ETFE膜在辐射下发生了 C-C键 的断裂. 在随后的工作中, Wang等人 ${ }^{[34]}$ 进一步优化 了辐射预处理和接枝聚合反应的条件. 用水作为稀 释溶剂, 代替了此前用的异丙醇. 这种方法不但降低 了电子束的辐射剂量(从70 kGy降低到30 40 kGy)和 对氯甲基苯乙烯的浓度 (从20\%降低到 5\%, 体积浓 度), 而且得到了高离子交换容量的阴离子交换膜 $(\mathrm{IEC}=2.10 \mathrm{mmol} / \mathrm{g})$.

全氟磺酸质子交换膜由于具有高的电导率和稳 定性, 被广泛用于质子交换膜燃料电池, 而全氟阴离 子交换膜的报道较少. Park等人 ${ }^{[35]}$ 以 $3 \mathrm{M}$ 公司的全氟 磺酰氟聚合物和全氟磺胺聚合物为原料, 通过磺酰 氟基团与胺基以及磺胺与卤代烃之间的反应, 将季 铵盐基团接枝到全氟聚合物主链上, 分别得到全氟 阴离子交换膜Gen 1 和Gen 2.

从上述制备方法可以看出, 功能单体共聚和高 分子接枝法制备聚烯烃类阴离子交换膜具有各自的 优缺点. 功能单体的成本较高, 需要经过复杂的分离 和提纯, 增加了膜制备的成本, 但是功能单体的共聚 能够精确控制膜的化学结构和组成, 有利于膜的结 构与性能的优化. 高分子接枝的方法相对容易, 成本 较低, 制作工艺简单, 有利于大规模工业化制备, 但 是辐射接枝对设备要求较高, 并且辐射容易对高分 子主链产生机械降解, 进而不利于燃料电池应用. 此 外, 如何采用辐射接枝精确控制膜的化学组成和批 次的稳定性也需要进一步研究.

\section{2 聚烯烃类阴离子交换膜的结构与性能}

\section{1 微相分离结构}

亲水/疏水的相分离结构有利于在阴离子交换膜 内形成贯通的离子传输通道, 进而提高膜的离子传导 率 $^{[36]}$. 目前, 人们通过合成嵌段型 ${ }^{[37,38]}$ 、梳状 ${ }^{[39,40]}$ 、离 子簇型 ${ }^{[41,42]}$ 和侧链型 ${ }^{[43,44]}$ 等化学结构规整的阴离子 交换膜来实现亲水/疏水的相分离结构(图3), 并且采 用透射电子显微镜(TEM)、原子力显微镜( AFM) 和小

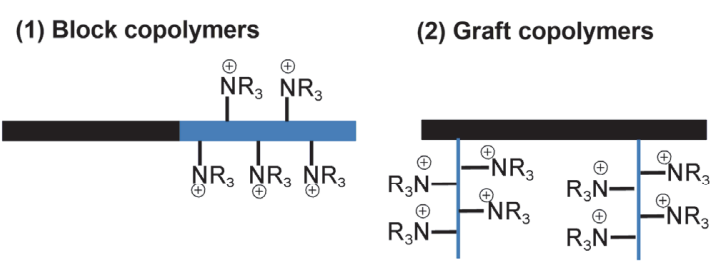

(3) Introduction of bulky hydrophobic groups

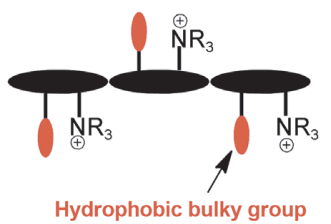

图 3 (网络版彩色)具有相分离的聚烯烃类阴离子交换膜的化学结构 示意图

Figure 3 (Color online) Illustrations of several polymer architectures of AEMs based on polyolefins with well-defined microphase-separated morphology

角X射线散射 (SAXS) 等技术研究阴离子交换膜的化 学结构与微观结构之间的关系, 其中离聚物峰 (ionomer peak)的出现和位置则是SAXS测试的重要 参数.

虽然采用ROMP方法具有较好的可控性，能够制 备结构有序的嵌段聚合物, 然而到目前为止, 还未见 用来制备嵌段型的阴离子交换膜. $\mathrm{Li}$ 等人 ${ }^{[45]}$ 通过丁二 烯与对氯甲基苯乙烯的阴离子共聚和随后的加氢反 应、季铵化反应, 得到嵌段型聚烯烃类阴离子交换膜. SAXS研究结果表明, 膜内具有规整的亲水/疏水的 相分离结构, $d$-spacing值在 $48.3 \sim 69.8 \mathrm{~nm}$, 并且湿度 对膜的相分离结构影响较小. 此外, 高角度环形暗场 扫描TEM测试同样验证了SAXS的结果, 并且观察到 典型的双连续相聚合物结构, 如图4所示.

尽管Ziegle-Natta催化剂催化的 $\alpha$-烯烃聚合也难 以制备结构可控的聚合物, 但是通过改变疏水链段 的疏水性来实现膜的相分离结构. 本课题组采用大 位阻的4-甲基-1-戊烯 ${ }^{[25]}$ 及 4-苯基-1-丁烯 ${ }^{[26]}$ 分别与 11-溴-1-十一碳烯(功能单体 10, 图2)共聚和季铵化反 应, 同样得到具有亲水/疏水相分离结构的聚烯烃类 阴离子交换膜. 此外, Zhu等人 ${ }^{[22]}$ 采用开环易位聚合 反应, 进行含有二茂钴离子的环辛烯功能单体5(图2) 与环辛烯的聚合, 得到侧链型的阴离子交换膜材料. $\mathrm{SAXS}$ 测试结果表明, 离子交换容量为 1.53 和 1.86 $\mathrm{mmol} / \mathrm{g}$ 的膜分别在 $q^{*}=1.28$ 和 $1.54 \mathrm{~nm}^{-1}$ 处有一个宽的 离聚物峰, 说明该聚烯烃阴离子交换膜具有明显的 相分离结构, 并且原子力显微镜也观察到了相分离 

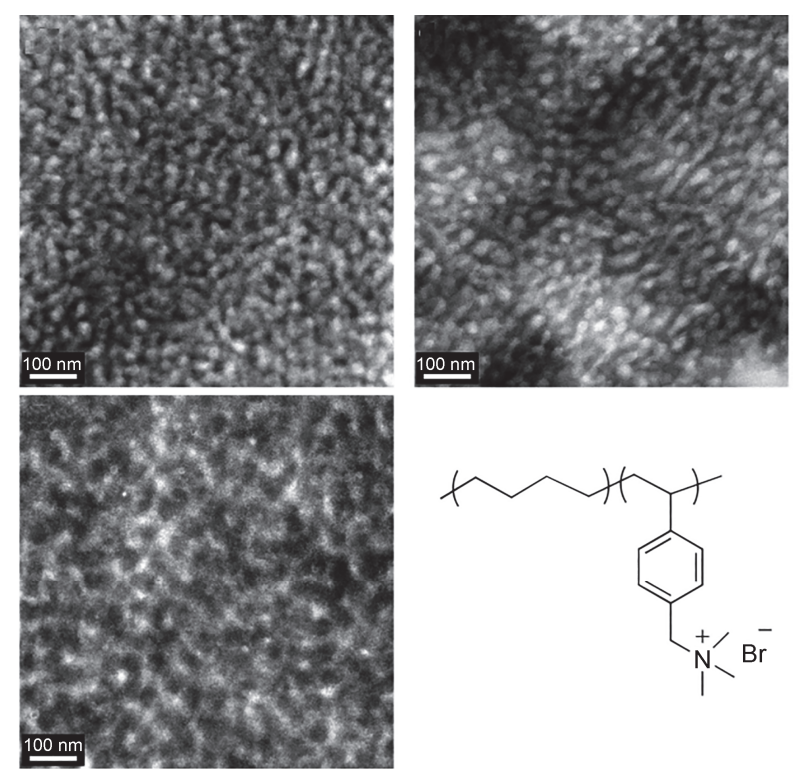

图 4 嵌段型阴离子膜的结构与 TEM图

Figure 4 Chemical structure and TEM images of block copolymers membrane

结构.

辐射接枝得到的梳状结构阴离子交换膜材料也 具有亲水/疏水的相分离结构. Ponce-González等人 ${ }^{[46]}$ 采用SAXS技术对比了不同阳离子基团的辐射接枝聚 烯烃类阴离子交换膜的微观结构. 在干态下, 含有哌 啶盐和吡咯盐阳离子基团的阴离子交换膜在 $q^{*}=0.22$ $\mathrm{nm}^{-1}$ 处出现一个离聚物峰, 而含有芐基三甲基季铵 盐的膜的离聚物峰则出现在 $q^{*}=0.16 \mathrm{~nm}^{-1}$ 处; 当测试 条件变为湿态时, 哌啶盐和吡咯盐的阴离子交换膜
的离聚物峰迁移到 $q^{*}=0.11 \mathrm{~nm}^{-1}$. 此外, 对这 3 种聚 烯烃阴离子交换膜在沸水中进行处理, 它们的离聚 物峰的位置不变.

\section{2 离子传导率}

离子传导率是阴离子交换膜的重要参数, 对碱 性燃料电池的性能起着至关重要的作用. 然而, 膜的 电导率受到很多因素的影响, 包括离子交换容量、吸 水率、相分离结构、阳离子基团的种类等. 通常来说, 高的IEC 和高的吸水率有利于离子的传输, 但是过高 的吸水率则使得膜的机械强度和膜内的离子浓度下 降, 离子传导率反而降低. 因此, 通过提高IEC来增 加聚烯烃阴离子交换膜的电导率往往伴随着膜溶胀 的急剧上升.

为了更好地比较各种结构的聚烯烃类阴离子交 换膜, 图 5 总结了膜的电导率和IEC以及膜的化学结 构之间的关系. 共价交联是一种控制膜溶胀、提高电 导率的有效方法. Robertson等人 ${ }^{[17]}$ 通过可交联的功 能单体 3 与环辛烯的ROMP反应, 得到交联的聚烯烃 阴离子交换膜, 并且室温下膜的氢氧根离子电导率 达到 $68.7 \mathrm{mS} / \mathrm{cm}$, 拉伸强度达到 $15.2 \mathrm{MPa}$. 本课题 组 ${ }^{[24]}$ 在 11-溴-1-十一碳烯 (功能单体 10, 图 2) 与丙烯 的共聚体系中, 引人可交联的苯乙烯单体(对-(3-丁 烯基)苯乙烯), 通过热交联的方法, 得到交联的阴离 子交换膜材料. 膜在IEC为 $1.65 \mathrm{mmol} / \mathrm{g}$ 时, 室温下氢 氧根离子电导率达到 $16.3 \mathrm{mS} / \mathrm{cm}$, 溶胀率仅为 $3.5 \%$.

通过嵌段聚合物的合成以及疏水段分子结构的
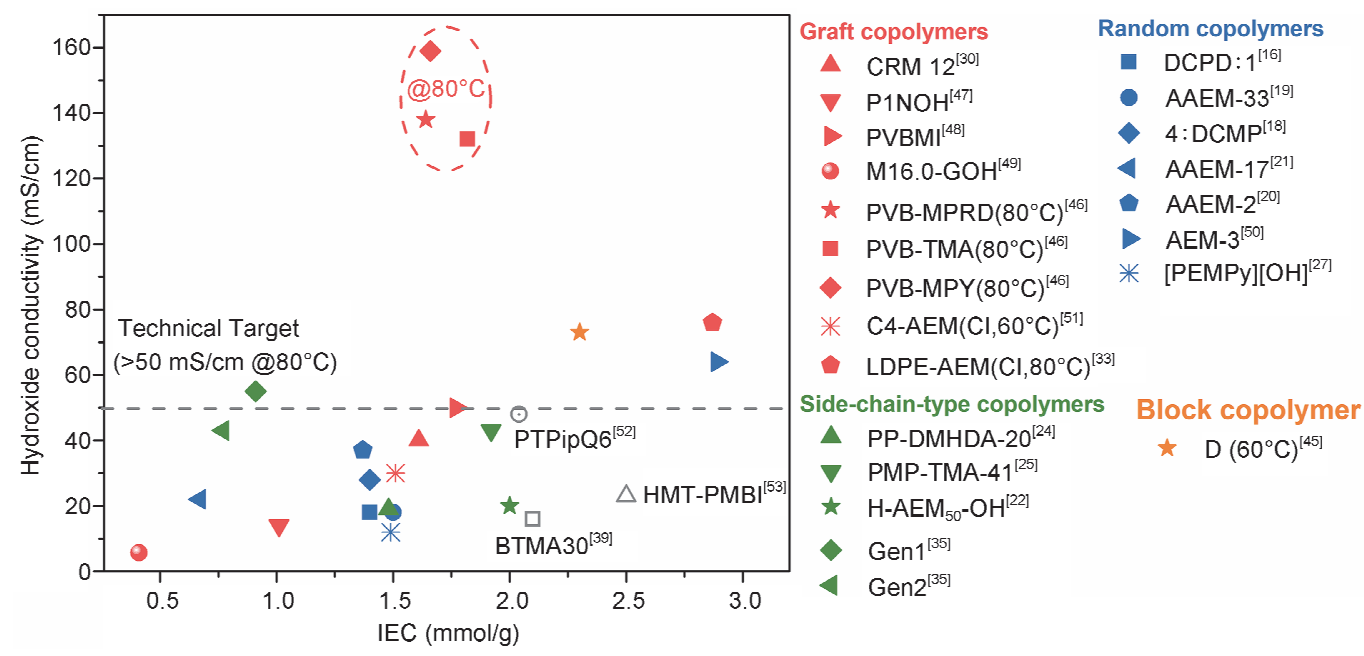

图 5 (网络版彩色) 氢氧根离子电导率 $\left(20 \sim 30^{\circ} \mathrm{C}\right)$ 与离子交换容量之间的关系

Figure 5 (Color online) Hydroxide conductivity at $20-30^{\circ} \mathrm{C}$ of AEMs as a function of IEC 
调控, 有利于膜内亲水/疏水相分离结构的形成, 从 而实现离子传导率的提高. $\mathrm{Li}$ 等人 ${ }^{[45]}$ 制备的嵌段型聚 烯烃阴离子交换膜 $\mathrm{D}$ 在高的离子交换容量 $(\mathrm{IEC}=2.30$ $\mathrm{mmol} / \mathrm{g}$ )下, 具有低的吸水率 ( $37 \%$, 质量百分数)和高 的氢氧根离子电导率 $\left(73 \mathrm{mS} / \mathrm{cm}, 60^{\circ} \mathrm{C}\right)$. 本课题组 ${ }^{[25]}$ 通过在聚烯烃阴离子交换膜中引人大位阻输水基团, 形成有序的亲水/疏水微相分离结构, 膜的氢氧根离 子电导率达到 $43.2 \mathrm{mS} / \mathrm{cm}(\mathrm{IEC}=1.9 \mathrm{mmol} / \mathrm{g})$.

辐射接枝制备的聚烯烃类阴离子交换膜具有较 好的抗溶胀性能, 通过前面所说的方法, 极大地提高 膜的IEC值(接枝率), 得到高电导率的阴离子交换膜. 通过辐射接枝得到的 PVB-TMA, PVB-MPY和PVBMPRD阴离子交换膜, 它们具有相同的主链结构和离 子含量, 但是阳离子基团不一样. 研究发现, 吡咯盐 的膜具有较高的氢氧根离子电导率, $80^{\circ} \mathrm{C}$ 下达到 159 $\mathrm{mS} / \mathrm{cm}^{[46]}$.

\section{3 碱性稳定性}

阴离子交换膜的碱性稳定性决定了碱性燃料电 池的使用寿命, 是目前研究的热点之一. 碱性稳定性 的研究方法包括研究电导率、离子交换容量、膜重量 等膜的物理参数随着稳定性测试时间的变化, 而降 解的机理则通过对比降解前后膜的各种谱图得出. 但是, 目前碱性稳定性测试的标准不统一, 主要体现 在测试的温度和碱液的浓度不统一. 由于烯烃聚合 物在碱性条件下较为稳定, 因此不同聚烯烃类阴离 子交换膜的碱性稳定性的差异则主要体现在阳离子 基团. 阳离子基团的碱性稳定性越高, 则对应的阴离 子交换膜稳定性越好. 图6列出聚烯烃类阴离子交换 膜的碱性稳定性测试条件和测试结果.

由于聚烯烃主链结构在碱性条件下的稳定性好, 因此, 含有碱稳定的阳离子基团的聚烯烃阴离子交 换膜具有高的碱性稳定性. 贲基三甲基季铵盐 (BTMA)基团是研究最多的阳离子, 但是含有BTMA 基团的聚苯醚和聚醚砜阴离子交换膜的碱性稳定性 较差 ${ }^{[9,10,39,41]}$. 有趣的是, 辐射接枝制备的BTMA型 聚烯烃类阴离子交换膜显示出较为优异的碱性稳定 性, 在 $1 \mathrm{~mol} / \mathrm{L} \mathrm{KOH}$ 和 $80^{\circ} \mathrm{C}$ 条件下, 7 和 $28 \mathrm{~d}$ 后, IEC分 别下降 $12 \%$ 和 $27 \%{ }^{[33,51]}$. 随后, 他们通过拉曼、IR等 技术研究其降解机理. 结果表明, 聚烯烃阴离子交换 膜中的BTMA基团受到氢氧根离子的进攻, 生成芐 醇结构, 使得离子交换容量下降 ${ }^{[33]}$. 为了进一步提

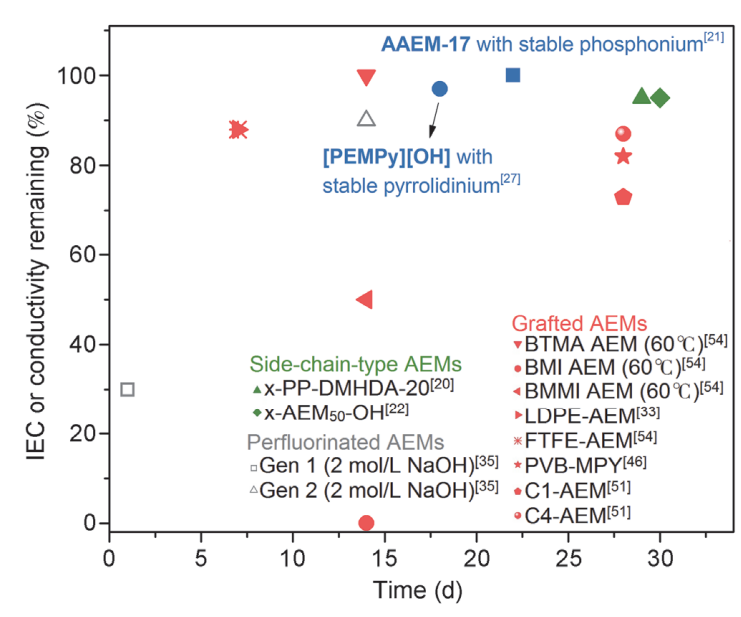

图 6 (网络版彩色) 聚烯烃类阴离子交换膜的碱性稳定性

Figure 6 (Color online) Alkaline stability of AEMs based on polyolefins

高碱性稳定性, 本课题组 ${ }^{[24]}$ 制备了侧链型聚烯烃阴 离子交换膜(x-PP-DMHDA-20), $10 \mathrm{~mol} / \mathrm{L} \mathrm{NaOH} 80^{\circ} \mathrm{C}$ 条件下测试700 h 后, 电导率仅仅下降 $3 \%$. 同时, 研 究人员将碱稳定的阳离子基团，包括位阻型季鏻

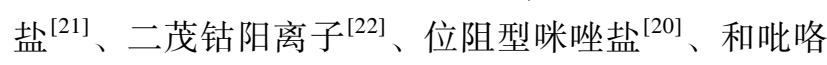
盐 ${ }^{[27]}$, 分别引入聚烯烃阴离子交换膜中, 得到高碱 性稳定性的膜材料. 例如, 含有稳定的位阻型季鏻盐 和金属离子的 AAEM-17 $7^{[21]}$ 和 $\mathrm{H}-\mathrm{AEM}_{50}-\mathrm{OH}^{[22]}$ 的聚烯 烃类阴离子交换膜具有较高的碱性稳定性, 能够在 1 $\mathrm{mol} / \mathrm{L} \mathrm{NaOH}$ 和 $80^{\circ} \mathrm{C}$ 的条件下, 分别稳定 $22 \mathrm{~d}$ 和 1 个月. 然而, 由于聚烯烃溶解性较差, 对功能基团的降解机 理的研究依然处在初级阶段, 并且碱性稳定性离美 国能源部(DOE)的要求 $(>5000 \mathrm{~h}$ )还有一定的差距.

聚合物主链对膜的碱性稳定性也有一定的影响. 同样是吡咯盐型聚烯烃类阴离子交换膜, 在 $1 \mathrm{~mol} / \mathrm{L}$ $\mathrm{NaOH}$ 和 $80^{\circ} \mathrm{C}$ 的测试条件下, 辐射接枝的吡咯盐型的 膜(PVB-MPY)测试 $28 \mathrm{~d}$ 后, IEC值下降 $18 \%{ }^{[46]}$; 而功 能单体共聚得到的膜([PEMPy][OH])在测试18 d 后, 电导率仅下降 $3 \%{ }^{[27]}$. 虽然聚乙烯辐射接枝的阴离子 交换膜(LDPE-AEM) 与聚 (乙烯-四氟乙烯)接枝的阴 离子交换膜(ETFE-AEM)的碱性稳定性相同(7 d, IEC 下降 $12 \%$ ), 但是ETFE-AEM膜的厚度下降 $30 \%$, 导致 降解后的膜机械强度下降 ${ }^{[33]}$.

\section{3 聚烯烃类阴离子交换膜的氢/氧燃料电池 性能}

阴离子交换膜燃料电池的性能与很多因素有关, 
包括膜的性能、催化剂的种类和含量、膜电极的制备 工艺、测试条件(温度、湿度、气体种类和流速等)、 黏结剂的性能等等. 这里总结了采用聚烯烃类阴离 子交换膜的氢/氧燃料电池性能, 并初步讨论上述影 响因素对燃料电池性能的影响.

表1列出了聚烯烃类阴离子交换膜燃料电池的初 始性能以及测试的条件. 目前, 辐射接枝的阴离子交 换膜在氢/氧燃料电池中的研究较多. Mamlouk等 人 $^{[32]}$ 报道了以低密度聚丙烯为聚合物主链, 通过辐 射接枝法得到阴离子交换膜X23, 该膜在氢/氧燃料 电池中最大功率密度能够达到 $823 \mathrm{~mW} / \mathrm{cm}^{2}$. PonceGonzález等人 ${ }^{[46]}$ 制备了含有不同阳离子基团的ETFE 接枝的阴离子交换膜. 当采用 $\mathrm{PtRu} / \mathrm{C}$ 作为阳极催化 剂时, 季铵盐型阴离子交换膜(PVB-TMA) 最大的功 率密度能够达到 $500 \mathrm{~mW} / \mathrm{cm}^{2}$, 当提高背压到 0.1 $\mathrm{MPa}$, 最大功率密度能够达到 $640 \mathrm{~mW} / \mathrm{cm}^{2}$. 由于吡 咯盐阴离子交换膜(PVB-MPY)具有较高的电导率, 它在燃料电池中的功率密度在 $0.1 \mathrm{MPa}$ 背压下能够达 到 $800 \mathrm{~mW} / \mathrm{cm}^{2}$. 当采用更薄的聚合物薄膜进行接枝 聚合, 吡咯盐型阴离子交换膜(E25-PVB-MPY)在0.1 $\mathrm{MPa}$ 背压下能够达到 $980 \mathrm{~mW} / \mathrm{cm}^{2}$. Wang等人 ${ }^{[56]}$ 采用 辐射接枝聚合物作为黏结剂, $\mathrm{PtRu} / \mathrm{C}$ 和 $\mathrm{Pt} / \mathrm{C}$ 分别为阳 极和阴极催化剂时, 季铵盐型ETFE接枝的阴离子交 换膜的氢/氧燃料电池性能能够达到 $1570 \mathrm{~mW} / \mathrm{cm}^{2}$. 值得一提的是, 当采用 $\mathrm{Ag} / \mathrm{C}$ 为阳极催化剂, 燃料电 池最大功率密度同样能够超过 $1000 \mathrm{~mW} / \mathrm{cm}^{2}$. Omasta
等人 ${ }^{[57]}$ 采用季铵盐型ETFE接枝的阴离子交换膜, 通 过调控阳极催化剂墨水中各组分的比例, 缓解了水 的传递对碱性燃料电池性能的限制, 将碱性然料电 池的最大功率密度提高到 $1900 \mathrm{~mW} / \mathrm{cm}^{2}$.

Park等人 ${ }^{[35]}$ 将制备的可溶的全氟阴离子传导聚 合物作为膜和黏结剂用于氢氧燃料电池中, 最大功 率密度能够达到 $300 \mathrm{~mW} / \mathrm{cm}^{2}$. 本课题组 ${ }^{[27]}$ 采用共聚 得到的聚烯烃类阴离子交换膜, 在氢/氧燃料电池中, 最大功率密度能够达到 $122 \mathrm{~mW} / \mathrm{cm}^{2}$.

\section{4 总结与展望}

综上所述, 虽然国内外学者对聚烯烃类阴离子 交换膜的制备和性能方面进行了广泛的研究, 但是 目前聚烯烃类阴离子交换膜的研究才刚刚起步, 这 主要是由于聚烯烃的合成方法较少, 导致膜的微结 构和化学结构的调控非常有限. 科研工作者需要经 过更广泛、深人的研究, 可以在以下 3 个方面实现突 破: (1) 新合成方法的开发. 正如前面所说, 由于聚 烯烃的溶解性问题, 导致功能基团的稳定性研究不 够. 因此, 需要开发新的合成方法, 制备可溶性的聚 烯烃，简化成膜的方法. 另外，新的合成方法可以调 控功能基团的结构, 有利于进一步研究阴离子交换 膜的降解机理; (2) 电池性能研究. 虽然辐射接枝法 制备的聚烯烃阴离子交换膜材料显示出优异的燃料 电池初始性能, 但是阴离子交换膜燃料电池的研究 才刚刚开始. 由于膜的碱性稳定性较差, 需要进一步

\section{表 1 碱性燃料电池初始性能}

Table $1 \mathrm{H}_{2} / \mathrm{O}_{2}$ fuel cell performance using AEMs based on polyolefins

\begin{tabular}{|c|c|c|c|c|}
\hline 样品 & 结构 & 测试条件 & 最高能量密度 $\left(\mathrm{mW} / \mathrm{cm}^{2}\right)$ & 文献 \\
\hline Membrane \# 2 & & $40^{\circ} \mathrm{C}, \mathrm{Pt}\left(2.0 \mathrm{mg} / \mathrm{cm}^{2}\right), \mathrm{H}_{2} / \mathrm{O}_{2}(100 \mathrm{ccm})$ & 48 & [31] \\
\hline $\mathrm{X}-23$ & & $60^{\circ} \mathrm{C}, \mathrm{Pt}\left(0.4 \mathrm{mg} / \mathrm{cm}^{2}\right), \mathrm{H}_{2} / \mathrm{O}_{2}(1 \mathrm{bar})$ & 823 & [32] \\
\hline PVBTMA & 辐 & $50^{\circ} \mathrm{C}, \mathrm{Pt}\left(0.4 \mathrm{mg} / \mathrm{cm}^{2}\right), \mathrm{H}_{2} / \mathrm{O}_{2}(0$ bar $)$ & $\sim 140$ & {$[48]$} \\
\hline $74.6 \%$ DOG & 射 & $50^{\circ} \mathrm{C}, \mathrm{Pt}, \mathrm{H}_{2} / \mathrm{O}_{2}$ & 608 & [55] \\
\hline E25-PVB-MPY & 接 & $60^{\circ} \mathrm{C}$, 阴极Pt/C, 阳极PtRu/C, 金属 $\left(0.4 \mathrm{mg} / \mathrm{cm}^{2}\right), \mathrm{H}_{2} / \mathrm{O}_{2}(400 \mathrm{ccm}, 0.1 \mathrm{MPa})$ & 980 & [46] \\
\hline ETFE-AEM & 枝 & $70^{\circ} \mathrm{C}$, 阴极PtRu/C, 金属 $\left(0.4 \mathrm{mg} / \mathrm{cm}^{2}\right), \mathrm{H}_{2} / \mathrm{O}_{2}(1000 \mathrm{ccm})$ & 1570(阴极Pt/C); 1110(阴极 Ag/C) & {$[56]$} \\
\hline LDPE-AEM & & 阴极Pt/C, 阳极PtRu/C, 金属 $\left(0.4 \mathrm{mg} / \mathrm{cm}^{2}\right), \mathrm{H}_{2} / \mathrm{O}_{2}(1000 \mathrm{ccm})$ & $960\left(60^{\circ} \mathrm{C}\right) ; 1450\left(80^{\circ} \mathrm{C}\right)$ & [33] \\
\hline ETFE-AEM & & $60^{\circ} \mathrm{C}$, 阴极Pt/C $\left(0.4 \mathrm{mg} / \mathrm{cm}^{2}\right)$, 阳极 $\mathrm{PtRu} / \mathrm{C}\left(0.71 \mathrm{mg} / \mathrm{cm}^{2}\right), \mathrm{H}_{2} / \mathrm{O}_{2}(1000 \mathrm{ccm})$ & 1900 & {$[57]$} \\
\hline PP-TMA-20 & 共 & $50^{\circ} \mathrm{C}, \mathrm{Pt}\left(0.5 \mathrm{mg} / \mathrm{cm}^{2}\right), \mathrm{H}_{2} / \mathrm{O}_{2}(500 \mathrm{ccm})$ & 122 & [24] \\
\hline PMP-TMA-41 & 聚 & $60^{\circ} \mathrm{C}, \mathrm{Pt}\left(0.5 \mathrm{mg} / \mathrm{cm}^{2}\right), \mathrm{H}_{2} / \mathrm{O}_{2}(200 \mathrm{ccm})$ & $\sim 40$ & {$[25]$} \\
\hline Gen 2 & $\begin{array}{l}\text { 全 } \\
\text { 氟 }\end{array}$ & $60^{\circ} \mathrm{C}, \mathrm{Pt}\left(0.4 \mathrm{mg} / \mathrm{cm}^{2}\right), \mathrm{H}_{2} / \mathrm{O}_{2}(1.2 \mathrm{slpm}, 121 \mathrm{kPa})$ & $\sim 300$ & [35] \\
\hline
\end{tabular}


加强电池性能和寿命的研究, 尤其是膜在电池环境 下的降解机理等等; (3) 由于聚烯烃的玻璃化转变温 度较低, 导致膜不能在高温条件下使用, 进一步限制
了燃料电池性能的提高. 相信随着研究人员的不断 研究, 符合燃料电池使用的聚烯烃类阴离子交换膜 会出现新的突破.

\section{参考文献}

1 Varcoe J R, Atanassov P, Dekel D R, et al. Anion-exchange membranes in electrochemical energy systems. Energy Environ Sci, 2014, 7: 3135-3191

2 Wang Y J, Qiao J, Baker R, et al. Alkaline polymer electrolyte membranes for fuel cell applications. Chem Soc Rev, 2013, 42: $5768-5787$

3 Gottesfeld S, Dekel D R, Page M, et al. Anion exchange membrane fuel cells: Current status and remaining challenges. J Power Sources, 2018, 375: 170-184

4 Cheng J, He G, Zhang F. A mini-review on anion exchange membranes for fuel cell applications: Stability issue and addressing strategies. Int J Hydrogen Energy, 2015, 40: 7348-7360

5 Merle G, Wessling M, Nijmeijer K. Anion exchange membranes for alkaline fuel cells: A review. J Membr Sci, 2011, 377: 1-35

6 Sun Z, Pan J, Guo J, et al. The alkaline stability of anion exchange membrane for fuel cell applications: The effects of alkaline media. Adv Sci, 2018, 5: 1800065

7 Marino M G, Kreuer K D. Alkaline stability of quaternary ammonium cations for alkaline fuel cell membranes and ionic liquids. ChemSusChem, 2015, 8: 513-523

8 Mohanty A D, Bae C. Mechanistic analysis of ammonium cation stability for alkaline exchange membrane fuel cells. J Mater Chem A, 2014, 2: 17314-17320

9 Pan J, Lu S, Li Y, et al. High-performance alkaline polymer electrolyte for fuel cell applications. Adv Func Mater, 2010, 20: 312-319

10 Yan J, Hickner M A. Anion exchange membranes by bromination of benzylmethyl-containing poly(sulfone)s. Macromolecules, 2010, 43: $2349-2356$

11 Ran J, Wu L, Ru Y, et al. Anion exchange membranes (AEMs) based on poly(2,6-dimethyl-1,4-phenylene oxide) (PPO) and its derivatives. Polym Chem, 2015, 6: 5809-5826

12 Si J, Lu S, Xu X, et al. A gemini quaternary ammonium poly(ether ether ketone) anion-exchange membrane for alkaline fuel cell: Design, synthesis, and properties. ChemSusChem, 2014, 7: 3389-3395

13 Thomas O D, Soo K J W Y, Peckham T J, et al. A stable hydroxide-conducting polymer. J Am Chem Soc, 2012, 134: 10753-10756

14 Arges C G, Ramani V. Two-dimensional NMR spectroscopy reveals cation-triggered backbone degradation in polysulfone-based anion exchange membranes. Proc Natl Acad Sci USA, 2013, 110: 2490-2495

15 Mohanty A D, Tignor S E, Krause J A, et al. Systematic alkaline stability study of polymer backbones for anion exchange membrane applications. Macromolecules, 2016, 49: 3361-3372

16 Clark T J, Robertson N J, Kostalik IV H A, et al. A ring-opening metathesis polymerization route to alkaline anion exchange membranes: Development of hydroxide-conducting thin films from an ammonium-functionalized monomer. J Am Chem Soc, 2009, 131: 12888-12889

17 Robertson N J, Kostalik IV H A, Clark T J, et al. Tunable high performance cross-linked alkaline anion exchange membranes for fuel cell applications. J Am Chem Soc, 2010, 132: 3400-3404

Zha Y, Disabb-Miller M L, Johnson Z D, et al. Metal-cation-based anion exchange membranes. J Am Chem Soc, 2012, 134: 4493-4496

Kostalik IV H A, Clark T J, Robertson N J, et al. Solvent processable tetraalkylammonium-functionalized polyethylene for use as an alkaline anion exchange membrane. Macromolecules, 2010, 43: 7147-7150

20 You W, Hugar K M, Coates G W. Synthesis of alkaline anion exchange membranes with chemically stable imidazolium cations: Unexpected cross-linked macrocycles from ring-fused ROMP monomers. Macromolecules, 2018, 51: 3212-3218

21 Noonan K J T, Hugar K M, Kostalik IV H A, et al. Phosphonium-functionalized polyethylene: A new class of base-stable alkaline anion exchange membranes. J Am Chem Soc, 2012, 134: 18161-18164

22 Zhu T, Xu S, Rahman A, et al. Cationic metallo-polyelectrolytes for robust alkaline anion-exchange membrane. Angew Chem Int Ed, 2018, 57: 2388-2392

23 Zhang M, Kim H K, Chalkova E, et al. New polyethylene based anion exchange membranes (PE-AEMs) with high ionic conductivity. Macromolecules, 2011, 44: 5937-5946 
24 Zhang M, Liu J, Wang Y, et al. Highly stable anion exchange membranes based on quaternized polypropylene. J Mater Chem A, 2015, 3: $12284-12296$

25 Zhang M, Shan C, Liu L, et al. Facilitating anion transport in polyolefin-based anion exchange membranes via bulky side chains. ACS Appl Mater Interfaces, 2016, 8: 23321-23330

26 Meng Z, Su Y, Wu Y, et al. Synthesis and properties of quaternized polyolefins with bulky poly(4-phenyl-1-butene) moieties as anion exchange membranes. J Membr Sci, 2017, 541: 244-252

27 Gu F, Dong H, Li Y, et al. Base stable pyrrolidinium cations for alkaline anion exchange membrane applications. Macromolecules, 2014, 47: 6740-6747

28 Qiu B, Lin B, Si Z, et al. Bis-imidazolium-based anion-exchange membranes for alkaline fuel cells. J Power Sources, 2012, 217: 329-335

29 Danks T N, Slade R C T, Varcoe J R. Alkaline anion-exchange radiation-grafted membranes for possible electrochemical application in fuel cells. J Mater Chem, 2003, 13: 712-721

30 Liu H, Yang S, Wang S, et al. Preparation and characterization of radiation-grafted poly(tetrafluoroethylene-co-perfluoropropyl vinyl ether) membranes for alkaline anion-exchange membrane fuel cells. J Membr Sci, 2011, 369: 227-283

31 Fang J, Yang Y, Lu X, et al. Cross-linked, ETFE-derived and radiation grafted membranes for anion exchange membrane fuel cell applications. Int J Hydrogen Energy, 2012, 37: 594-602

32 Mamlouk M, Horsfall J A, Williams C, et al. Radiation grafted membranes for superior anion exchange polymer membrane fuel cells performance. Int J Hydrog Energy, 2012, 37: 11912-11920

33 Wang L, Brink J J, Liu Y, et al. Non-fluorinated pre-irradiation-grafted (peroxidated) LDPE-based anion-exchange membranes with high performance and stability. Energy Environ Sci, 2017, 10: 2154-2167

34 Wang L, Magliocca E, Cunningham E L, et al. An optimized synthesis of high performance radiation-grafted anion-exchange membranes. Green Chem, 2017, 19: 831-843

35 Park A M, Owczarczyk Z R, Garner L E, et al. Synthesis and characterization of perfluorinated anion exchange membranes. ECS Trans, 2017, 80: 957-966

36 Li N, Guiver M D. Ion transport by nanochannels in ion-containing aromatic copolymers. Macromolecules, 2014, 47: 2175-2198

37 Mohanty A D, Ryu C Y, Kim Y S, et al. Stable elastomeric anion exchange membranes based on quaternary ammonium-tethered polystyrene- $b$-poly(ethylene-co-butylene)- $b$-polystyrene triblock copolymers. Macromolecules, 2015, 48: 7085-7095

38 Tanaka M, Fukasawa K, Nishino E, et al. Anion conductive block poly(arylene ether)s: Synthesis, properties, and application in alkaline fuel cells. J Am Chem Soc, 2011, 133: 10646-10654

39 Li N, Leng Y, Hickner M A, et al. Highly stable, anion conductive, comb-shaped copolymers for alkaline fuel cells. J Am Chem Soc, 2013, 135: 10124-10133

40 Wang J, Wang X, Zu D, et al. N3-adamantyl imidazolium cations: Alkaline stability assessment and the corresponding comb-shaped anion exchange membranes. J Membr Sci, 2018, 545: 116-125

41 Weiber E A, Jannasch P. Ion distribution in quaternary-ammonium-functionalized aromatic polymers: Effects on the ionic clustering and conductivity of anion-exchange membranes. ChemSusChem, 2014, 7: 2621-2630

42 Li Q, Liu L, Miao Q, et al. A novel poly(2,6-dimethyl-1,4-phenylene oxide) with trifunctional ammonium moieties for alkaline anion exchange membranes. Chem Commun, 2014, 50: 2791-2793

43 Dang H S, Jannasch P. Exploring different cationic alkyl side chain designs for enhanced alkaline stability and hydroxide ion conductivity of anion-exchange membranes. Macromolecules, 2015, 48: 5742-5751

44 Liu L, Chu X, Liao J, et al. Tuning the properties of poly(2,6-dimethyl-1,4-phenylene oxide) anion exchange membranes and their performance in $\mathrm{H}_{2} / \mathrm{O}_{2}$ fuel cells. Energy Environ Sci, 2018, 11: 435-446

45 Li Y, Liu Y, Savage A M, et al. Polyethylene-based block copolymers for anion exchange membranes. Macromolecules, 2015, 48: 6523-6533

46 Ponce-González J, Whelligan D K, Wang L, et al. High performance aliphatic-heterocyclic benzyl-quaternary ammonium radiationgrafted anion-exchange membranes. Energy Environ Sci, 2016, 9: 3724-3735

$47 \mathrm{Hu}$ J, Zhang C, Cong J, et al. Plasma-grafted alkaline anion-exchange membranes based on polyvinyl chloride for potential application in direct alcohol fuel cell. J Power Sources, 2011, 196: 4483-4490

48 Deavin O I, Murphy S, Ong A L, et al. Anion-exchange membranes for alkaline polymer electrolyte fuel cells: Comparison of pendent benzyltrimethylammonium- and benzylmethylimidazolium-head-groups. Energy Environ Sci, 2012, 5: 8584-8597

49 Sherazi T A, Zahoor S, Raza R, et al. Guanidine functionalized radiation induced grafted anion-exchange membranes for solid alkaline fuel cells. Int J Hydrog Energy, 2015, 40: 786-796 
50 Wang C, Mo B, He Z, et al. Crosslinked norbornene copolymer anion exchange membrane for fuel cells. J Membr Sci, 2018, 556: $118-125$

51 Ponce-González J, Ouachan I, Varcoe J R, et al. Radiation-induced grafting of a butyl-spacer styrenic monomer onto ETFE: The synthesis of the most alkali stable radiation-grafted anion-exchange membrane to date. J Mater Chem A, 2018, 6: 823-827

52 Olsson J S, Pham T H, Jannasch P. Poly(arylene piperidinium) hydroxide ion exchange membranes: Synthesis, alkaline stability, and conductivity. Adv Funct Mater, 2018, 28: 1702758-1702767

53 Wright A G, Fan J, Britton B, et al. Hexamethyl-p-terphenyl poly(benzimidazolium): A universal hydroxide-conducting polymer for energy conversion devices. Energy Environ Sci, 2016, 9: 2130-2142

54 Page O M M, Poynton S D, Murphy S, et al. The alkali stability of radiation-grafted anion-exchange membranes containing pendent 1-benzyl-2,3-dimethylimidazolium head-groups. RSC Adv, 2013, 3: 579-587

55 Espiritu R, Mamlouk M, Scott K. Study on the effect of the degree of grafting on the performance of polyethylene-based anion exchange membrane for fuel cell application. Int J Hydrog Energy, 2016, 41: 1120-1133

56 Wang L, Brink J J, Varcoe J R. The first anion-exchange membrane fuel cell to exceed $1 \mathrm{~W} / \mathrm{cm}^{2}$ at $70^{\circ} \mathrm{C}$ with a non-Pt-group $\left(\mathrm{O}_{2}\right)$ cathode. Chem Commun, 2017, 53: 11771-11773

57 Omasta T J, Park A M, LaManna J M, et al. Beyond catalysis and membranes: Visualizing and solving the challenge of electrode water accumulation and flooding in AEMFCs. Energy Environ Sci, 2018, 11: 551-558 


\title{
Recent development in polyolefin-based anion exchange membrane for fuel cell application
}

\author{
Lei Liu, Xiaomeng Chu \& Nanwen $\mathrm{Li}^{*}$ \\ State Key Laboratory of Coal Conversion, Institute of Coal Chemistry, Chinese Academy of Sciences, Taiyuan 030001, China \\ * Corresponding author, E-mail: linanwen@sxicc.ac.cn
}

Anion exchange membrane (AEM) plays a critical role in many environmental and energy devices and processes, such as fuel cell, redox flow battery, and electrodialysis. In particular, when polymeric AEMs are exploited in alkaline fuel cells to replace liquid electrolytes, AEM fuel cells (AEMFC) have attracted worldwide attention because of several inherent advantages over proton exchange membrane fuel cells, e.g. the utilization of less expensive metallic catalysts and enhanced kinetics of oxygen reduction. Among different kinds of AEMs, polyolefin-based AEMs showed great potential for large-scale commercialization because of their excellent chemical stability, easy processability, and low cost.

The strategies to synthesize polyolefin anion exchange membrane materials included direct (co)polymerization with functionalized monomers and post-modification of polyolefins. Generally, the ring-opening metathesis polymerization and Ziegler-Natta catalyst mediated polymerization techniques have been successfully employed to synthesized polyolefin AEMs, using the functional monomers including the $\alpha$-olefin, norbornene, and cyclooctene. Therefore, the chemical structure and composition can be readily tuned to achieve the optimized properties of the resulting AEMs. However, the functional monomers that need complex synthetic procedure have greatly hampered its further commercialization. Radiation-grafting polymerization of vinylbenzyl chloride (VBC) on the polyolefin backbone has been confirmed as another effective method to produce AEMs. Both (partially or fully) fluorinated and hydrocarbon-based polyolefin have been grafted with poly(VBC) under high energy radiation (such as commercial electron-beam accelerators or ${ }^{60} \mathrm{Co} \gamma$-ray facilities), and subsequent quaternization allow the synthesis of AEMs with different ionic head-group. In order to produce high-performance AEMs, high irradiation doses were used to get high degree of grafting, which in turn results in a detrimental reduction in the mechanical properties of the resulting AEMs. Therefore, it is necessary to optimise the conditions of grafting reactions.

The physicochemical properties of polyolefin-based AEMs are investigated in detail by the measurement of ion exchange capacity, water uptake, swelling ratio, and ionic conductivity, which are well corelated to their chemical structure and microstructure. Moreover, $\mathrm{H}_{2} / \mathrm{O}_{2}$ AEMFC assembled from polyolefin-based AEMs (especially for radiation-grafted AEMs) are tested under various conditions in terms of types of electrochemical catalysts, cell temperature, gas flow rate, and so on.

Thus, this review summarized the recent developments of polyolefin-based AEMs from the synthetic methods to the properties of AEMs and their performance in $\mathrm{H}_{2} / \mathrm{O}_{2}$ fuel cell. The relationship between chemical structure, properties and morphology of polyolefin-based AEMs will be discussed. At last, we extend the discussion of AEMs to their performance in $\mathrm{H}_{2} / \mathrm{O}_{2}$ fuel cell, as dramatic improvements of AEM fuel cell performance is accomplished when using highperformance polyolefin-based AEMs.

polyolefin, anion exchange membrane, alkaline fuel cell, ionic conductivity, alkaline stability

doi: 10.1360/N972018-00880 